\title{
ANALYZING NONCOMBATANT EVACUATION OPERATIONS USING DISCRETE EVENT SIMULATION
}

\author{
Dallas Kuchel \\ Center for Army Analysis \\ 6001 Goethals Road \\ Fort Belvoir, Virginia 22060, USA
}

\begin{abstract}
Large scale evacuations can be extremely complex, requiring tremendous coordination and logistical support. Noncombatant Evacuation Operations (NEOs) present additional challenges of civil unrest and violence that congests the transportation network and can require military assistance to execute the evacuation. NEOs contain many moving parts and simultaneous processes including thousands of evacuees, vehicles, aircraft, and personnel tracking technology. Discrete event simulation is a technique well suited to handle the complex interactions between the entities and to analyze the behavior of the system. This paper describes the methodology used to analyze NEO by the Center for Army Analysis (CAA) and presents a case study that illustrates how modeling can be used to evaluate various courses of action and support decision making. When preparing to execute a NEO, decision makers use simulation modeling and analysis to evaluate evacuation timelines, allocate resources and lift assets, select safe haven locations, and determine support requirements for evacuees.
\end{abstract}

\section{INTRODUCTION}

There are millions of US citizens living in countries all around the world. The Department of State (DOS) is responsible for the well-being of US citizens abroad. In the event of an emergency, DOS will advise US citizens to evacuate the region and, if necessary, provide transportation. However in some instances, civil unrest and violence prevents US citizens from safely evacuating the region via commercial transportation. DOS may request military assistance from Department of Defense (DOD) in the form of a Noncombatant Evacuation Operation (NEO) to safely evacuate American citizens and designated host nation and third country nationals. (Joint Chiefs of Staff 2007).

Since 1988, DOS has conducted 315 evacuations abroad. Of these, 29 have required military assistance, with an average of at least one per year. Recent military assisted evacuation operations include Libya, Haiti, Guinea, and Lebanon (State 2013). In Lebanon after the outbreak of war in 2006, over 15,000 American citizens were evacuated over three weeks on flights arranged by DOD (Ford 2007).

Large scale evacuations are very complex and require tremendous logistical coordination. DOD faces additional challenges in uncertain resource levels and complex system tradeoffs. The Center for Army Analysis has helped Combatant Commands understand, analyze, and plan for NEO. Analysis helps determine evacuation timelines, evaluate location infrastructure, allocate resources, and understand system tradeoffs.

Discrete event simulation is a useful tool for this type of capacitated network analysis. In particular, simulation is useful for comparing courses of action and showing where queues form (Gregg 2010). It is easy to change an input variable and re-run the model. The NEO simulation model run takes only seconds to complete and output graphs are created automatically through Microsoft Excel output file allowing for rapid comparison and evaluation. 
According to Gregg (2010), "each instance of an evacuation operation is always a unique occurrence". Therefore, extending conclusions from an analysis of one NEO to another is not necessarily accurate. Thus, our approach is centered around creating an approach that can be quickly adapted and used to analyze a new NEO scenario and provide analysis to the decision maker in a matter of days. Often the Commander is concerned with quickly finding an acceptable solution, rather than taking additional time to find an optimal solution (Junkins 2012).

The objectives of this analysis are to minimize evacuation time, maximize resource utilization, and to identify limiting factors and choke points. In working with US Army Africa and USPACOM, decision makers find this information incredibly valuable when refining NEO plans, allocating resources, and determining support requirements during an evacuation.

\section{MODELING NEO ELEMENTS}

The typical NEO evacuation structure involves Assembly Areas (AAs), Evacuation Control Centers (ECCs), Temporary Safe Havens (TSH), and repatriation sites. Noncombatant evacuees (NCEs) arrive at AAs, where they come under DOD control. They are transported to an ECC, where they are processed into the NEO Tracking System (NTS). The NTS is used to account for NCEs throughout the evacuation process and to construct flights manifests. NCEs are then transported to a safe haven location that is outside of the area of risk. Safe and expedient evacuation from the area of risk is the primary objective and resources are allocated to minimize this evacuation time. A queue at the safe haven location is much preferred to a queue at the AAs or ECCs, which are inside the area of risk. Lastly, the NCEs are transported to a repatriation site, typically a major city like London, Paris, or a US city.

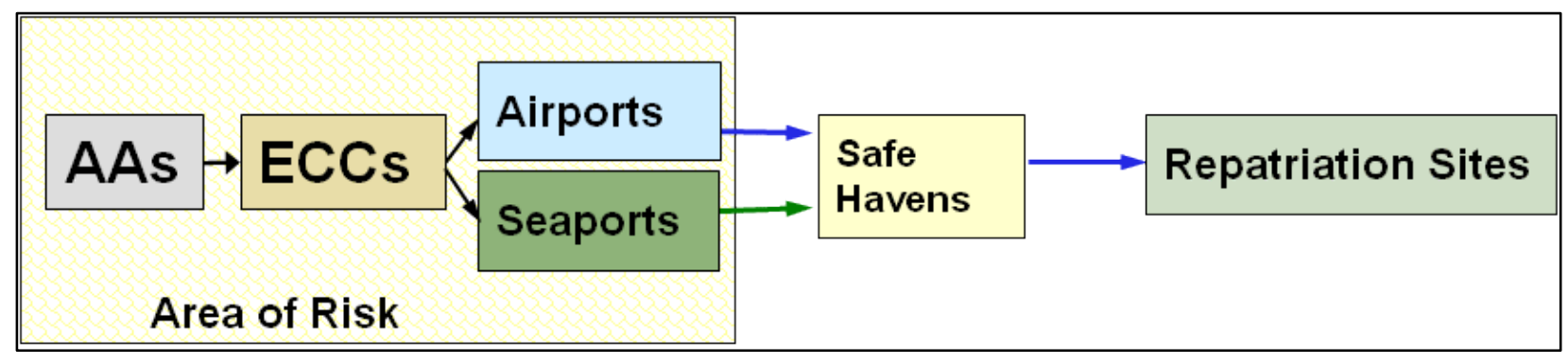

Figure 1: NEO Structure.

The following sections describe the approach used to model the NEO elements. Modeling was centered around creating reusable model logic modules that could be repurposed and adapted to allow for rapid development of scenarios. Modules were created for AAs, ECCs, TSHs, and repatriation. If a new AA is needed, then the modeler can simply copy and paste the AA module and rename variables and nodes. The following sections will discuss the elements that make up a module.

\subsection{Assembly Areas}

Assembly areas are normally located in high population density areas. They are typically locations that American citizens can reach on their own, without DOD provided transportation. Thus, there is an arrival process by which the NCEs appear at an AA. According to DOS, NCEs arrive over time with a bell curve skewed to the right, meaning that the arrival rate starts slow, then speeds up, and slows to completion. The arrival process is modeled as a Poisson process with inter-arrival times distributed exponentially. The rate of the arrival process varies over time to reflect the bell-shaped nature of the arrivals. Due to the stochastic nature of the arrival processes, 20 replications are run in the simulation and average statistics are utilized. 
Assembly areas are usually the least secure location in the evacuation process. They are often a building or compound located in an urban area such as an American Embassy or school. It is desirable to quickly move NCEs to the ECC, which is better able to handle large quantities of people and a better location at which to provide security. Helicopters or buses are often used to transport NCEs from the AAs to the ECC.

The approach used to model an AA is shown in Figure 2. To model an AA, we start with the arrival of NCEs. In block one of Figure 3, NCEs are created, a variable is updated to reflect the new number of NCEs, and the NCEs are stored in the transport queue. The NCEs will wait in the FIFO queue until an asset has arrived with sufficient capacity. Block 3 models the arrival of lift assets. Upon arrival, the asset will load up to its capacity and wait for a specified loading time. Block 4 contains logic for loading the asset, modifying variables to account for the transfer of NCEs, and delaying the asset by a load time. The asset then moves to the next destination, which in this case, is an ECC.

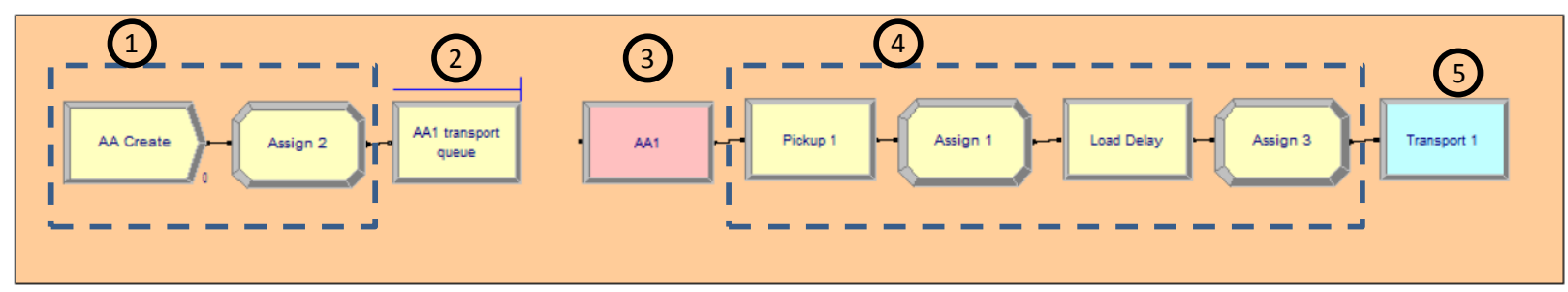

Figure 2: Assembly Area Module.

\subsection{Evacuation Control Centers}

ECCs conduct processing, screening, and logistic functions associated with emergency evacuation. ECCs are typically collocated with an airfield or seaport to enable rapid evacuation and are generally close to the AAs that feed into it. While ECCs are often still in the area of risk, they are more organized and secure than AAs and are better suited to handle large groups of NCEs. The primary functions of an ECC are accuracy, security, and speed (Joint Chiefs of Staff 2007).

Before NCEs can be routed onward, they must be registered into the NEO tracking system to maintain accountability for all NCEs and construct flight manifests. NTS processing is often an overlooked constraint during the evacuation process. An NTS machine consists of a computer system and an operator. The operator must gather proof of citizenship and documentation from the NCEs before they are cleared for transportation. Empirical data from USPACOM exercises has shown that initial entry into the system takes around seven minutes per person, or conversely, a machine can process nine NCEs per hour. With limited quantities of NTS machines, it is easy to see this process delaying evacuation.

Figure 3 shows the approach used to model an ECC. When an asset arrives, block one decides whether the asset is there to drop off passengers or to load passengers. The top route shows the logic used for unloading assets. After an unloading delay, the NCEs are dropped off and separated from the asset. The NCE entities proceed to block five and are processed into the NTS with a Seize, Delay, Release block with a specified number of resources. This allows us to capture the process of occupying a machine and the time delay associated with NTS processing. The quantity of NTS machines available is a decision point and can be easily adjusted in the model. The NCEs are then passed into a queue to await transportation to a TSH.

After the drop-off, the empty lift asset proceeds to block three. The lift asset may or may not be refueled, then is routed back to pick up more passengers. Assets can either always return to the location they came from or more complex logic can be used to compare the number of passengers in various queues and select the next location to pick up passengers. 
If the asset arrived empty, it is sent to block 6. The asset must first seize a unit of MOG before it can pick up passengers. The asset experiences a loading delay in block 7 as it loads NCEs, then releases the unit of MOG and transports to the destination.

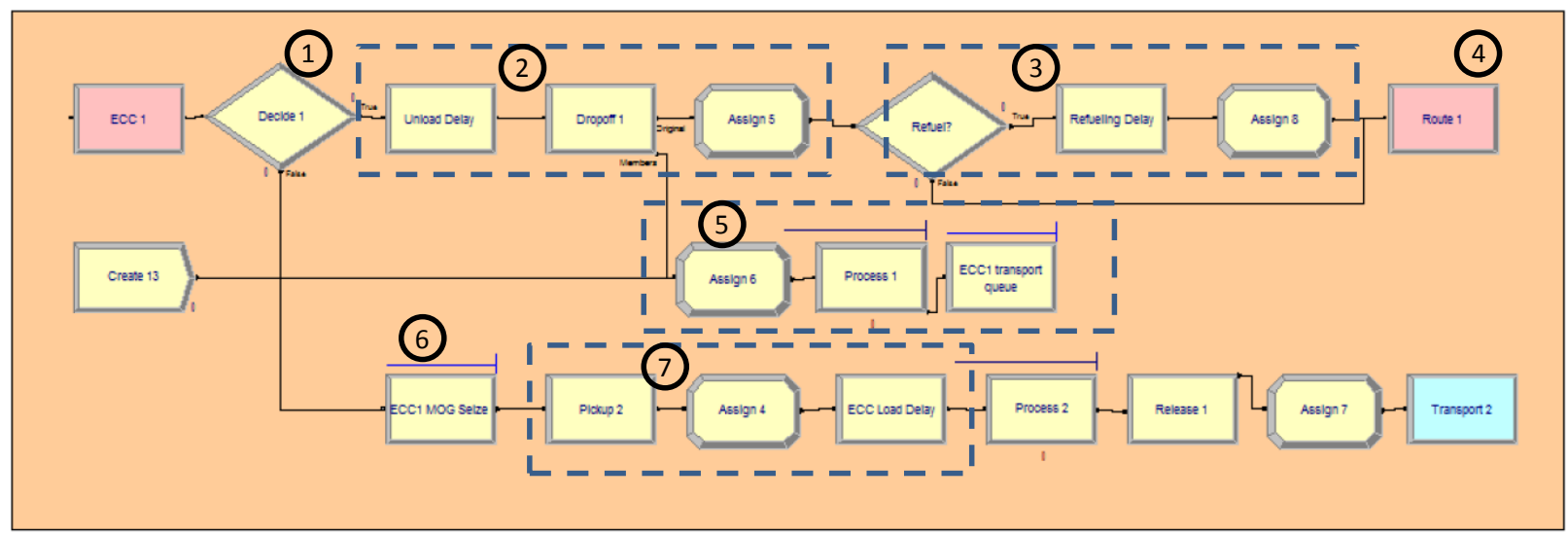

Figure 3: Evacuation Control Center Module.

\subsection{Temporary Safe Havens}

A TSH is a secure location to which NCEs can be moved quickly and easily. A nearby location is desired to enable rapid evacuation of the ECC. Safe havens will often need resources like hotels, stadiums, and other locations where NCEs can stay until transportation to the United States is available (Joint Chiefs of Staff 2007).

NCEs must be accounted for in the NTS system at safe havens, as well. However, after initial entry into the system NCEs process at a rate of 120 NCEs per hour. Therefore, only one or two NTS machines are needed at TSH locations.

When an asset arrives at a TSH, the asset must first obtain a unit of MOG to land. Block two determines whether the asset is there to unload passengers or pick-up passengers. If the asset is unloading, it drops off the passengers, who then process to block 5 which models processing into the NTS system through a seize-delay-release block. The NCEs then proceed to a queue awaiting transportation to repatriation. The empty asset proceeds to block 4 where it refuels and is sent back to pick up passengers.

If the asset arrives empty, then it was requested to pick up passengers awaiting lift. The asset is sent to block 6, where it requests MOG, loads NCEs, waits the required loading time, releases the unit of MOG, and travels to the destination.

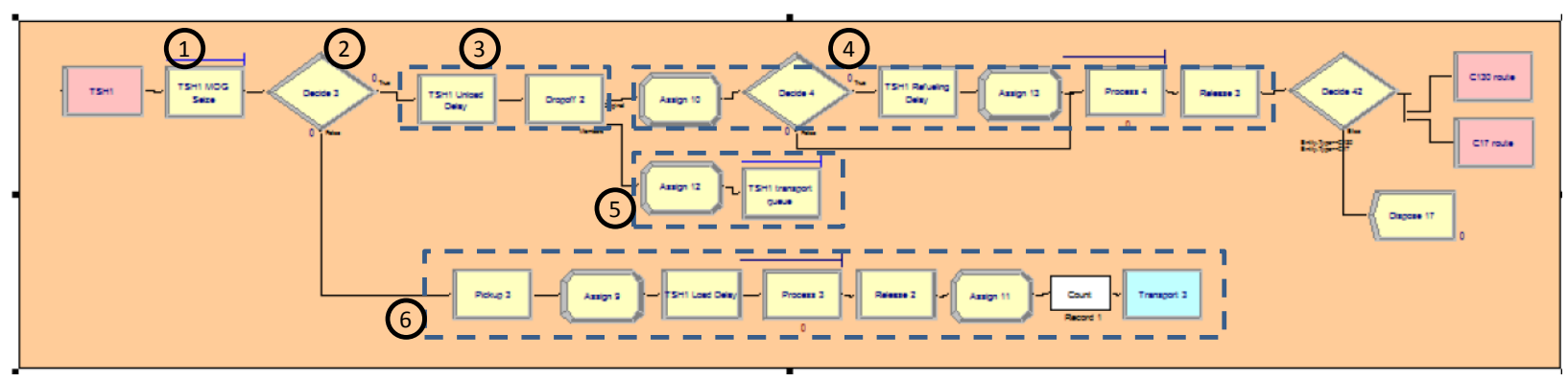

Figure 4: Temporary Safe Haven Module. 


\subsection{Repatriation Sites}

Repatriation is process by which American citizens are processed back into the United States of America after being evacuated from overseas. The Army is the executive agent for repatriation of NCEs (HQDA 2005).

To model repatriation sites, we simply receive the incoming lift asset, unload the passengers, and dispose the entities from the system. We track when the entities exit the system for analysis. The lift asset then returns to pickup more passengers.

\subsection{Routing Lift Assets}

Routing and allocating lift assets is a significant analytical problem in and of itself. CAA employs a mixed-integer program to help inform asset routing decisions. However, in this simulation, simplifying assumptions are made for modeling ease. When an aircraft unloads passengers, it must select the next location it will pick-up new passengers. This decision is made by comparing the number of NCEs in each queue. The asset will be routed to pick-up passengers from the location with the largest queue. An alternative routing logic would be to assign a set path to each asset. The asset would then repeat that assignment until all NCEs had been transported.

\section{NOTIONAL SCENARIO}

The following notional scenario demonstrates the manner in which the NEO simulation model has been used for real world NEO analysis. As shown in Figure 5, the operation is conducted in three phases. Area 1 is evacuated first, followed by Area 2, then Area 3. Area 1 consists of four locations. In this case, AA1 contains 10,000 NCEs, while ECCs 2, 3, and 4 each contain 1,000 NCEs. NCEs are transported via military airlift from the ECCs, which are co-located with airfields, to TSH1. The diagram also depicts the MOG of each airfield and the type of airlift that is used on each segment of the network.

Area 2 contains 15,000 NCEs and will begin evacuation on day 5. NCEs arrive at AA5 and are transported to ECC5 via ground transportation and rotary-wing lift with UH-60s and $\mathrm{CH}-47 \mathrm{~s}$. From ECC5, NCEs are flown via C-17 to TSH2.

Area 3 contains 12,000 NCEs. The NCEs will arrive at AA6 on day 11. NCEs are then transported via ground transportation to the nearby ECC6 where they are processed and flown to TSH1.

From the TSHs, NCEs are flown via CRAF (commercial air) to the repatriation site. 


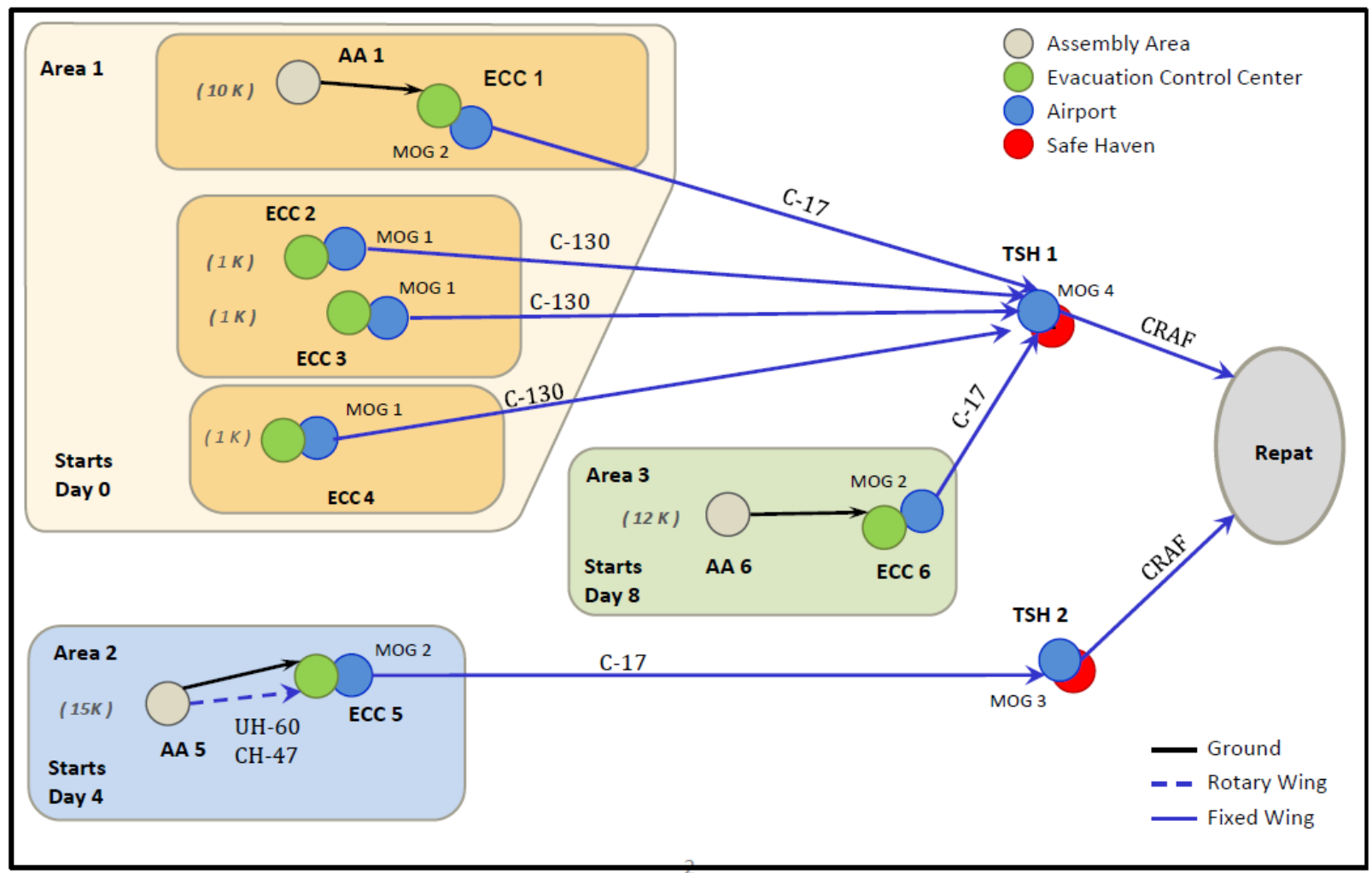

Figure 5: Notional NEO Scenario.

The evacuation is conducted in phases due to the large number of NCEs and limited resources (security forces, lift assets, NTS machines). After evacuation of Area 1, resources must be relocated to area 2. Evacuation of Area 2 is scheduled to begin on day 5 to allow for transportation and redistribution of resources. The distribution of NTS machines is shown in Table 1.

Table 1: NTS Machine Distribution.

\begin{tabular}{|c|c|c|c|c|c|c|c|c|c|c|c|}
\hline Location & AA1 & ECC1 & ECC2 & ECC3 & ECC4 & AA5 & ECC5 & AA6 & ECC6 & TSH1 & TSH2 \\
\hline Qty NTS Machines & 0 & 10 & 2 & 2 & 2 & 0 & 11 & 0 & 10 & 3 & 3 \\
\hline
\end{tabular}

\section{$4 \quad$ NOTIONAL OUTPUT AND INSIGHTS}

The simulation allows us to track individual NCEs throughout the system. We can keep track of the number of NCEs at each location over time. This information is extremely valuable to decision makers and NEO planners. The quantity of NCEs and duration they are held at a location determines the requirements for security forces, food, water, beds, and many other items.

Figure 6 shows the number of NCEs in Areas 1,2, and 3 over time. Evacuation of Area 1 starts slow and speeds up as additional lift assets become available. In this scenario, lift assets are phased in over time as they become available. Area 1 takes around 5.5 days to fully evacuate in this scenario.

On day 4, NCEs arrive at Area 2. Evacuation of Area 2 takes around 4 days and finishes on day 8. NCEs arrive to Area 3 on day 8. Evacuation of Area 3 takes around 4 days and finishes on day 12. 
Kuchel

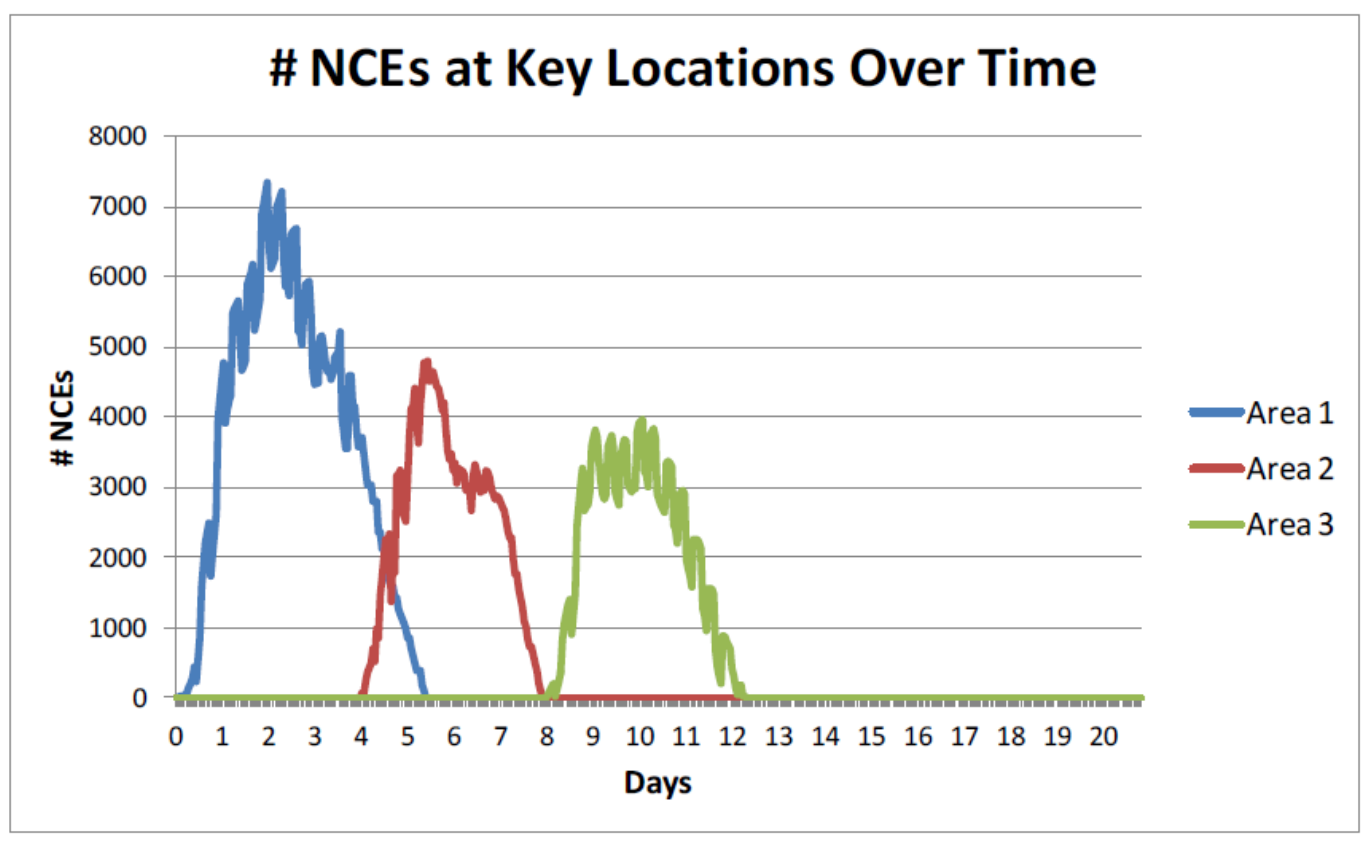

Figure 6: Area Contents Over Time.

It is often desirable to know where NCEs are within each area. Figure 7 shows AAs and ECCs 1, 5, and 6. We see that as the number of NCEs in AA1 decrease, the amount in ECC1 increases. We also see that ECC1 reaches a maximum quantity of around 5,000 NCEs and carries a quantity greater than 4,000 for days 2-4. AA1 is fully evacuated by day 4 and ECC 1 is fully evacuated by day 5.5 .

We see that AA5 is fully evacuated by day 7 but ECC5 is not fully evacuated until day 8. Rapid evacuation of the AA is desired as AA locations are typically more dangerous than ECC locations. We see that ECC5 reaches a maximum quantity near 5,000 NCEs on day 5 and contains at least 3,000 NCEs for days5, 6, and 7 .

AA6 receives NCEs on day 8 and does not finish evacuating until day 12 . We also note that ECC6 never realizes an accumulation of NCEs. There is always enough lift to transport NCEs out of ECC6 as quickly as they enter. ECC6 is fully evacuated on day 12, right after AA6 finishes evacuating. Therefore, AA6 constrains the overall evacuation of Area 3. Additional ground vehicles are needed if a faster evacuation is desired. 


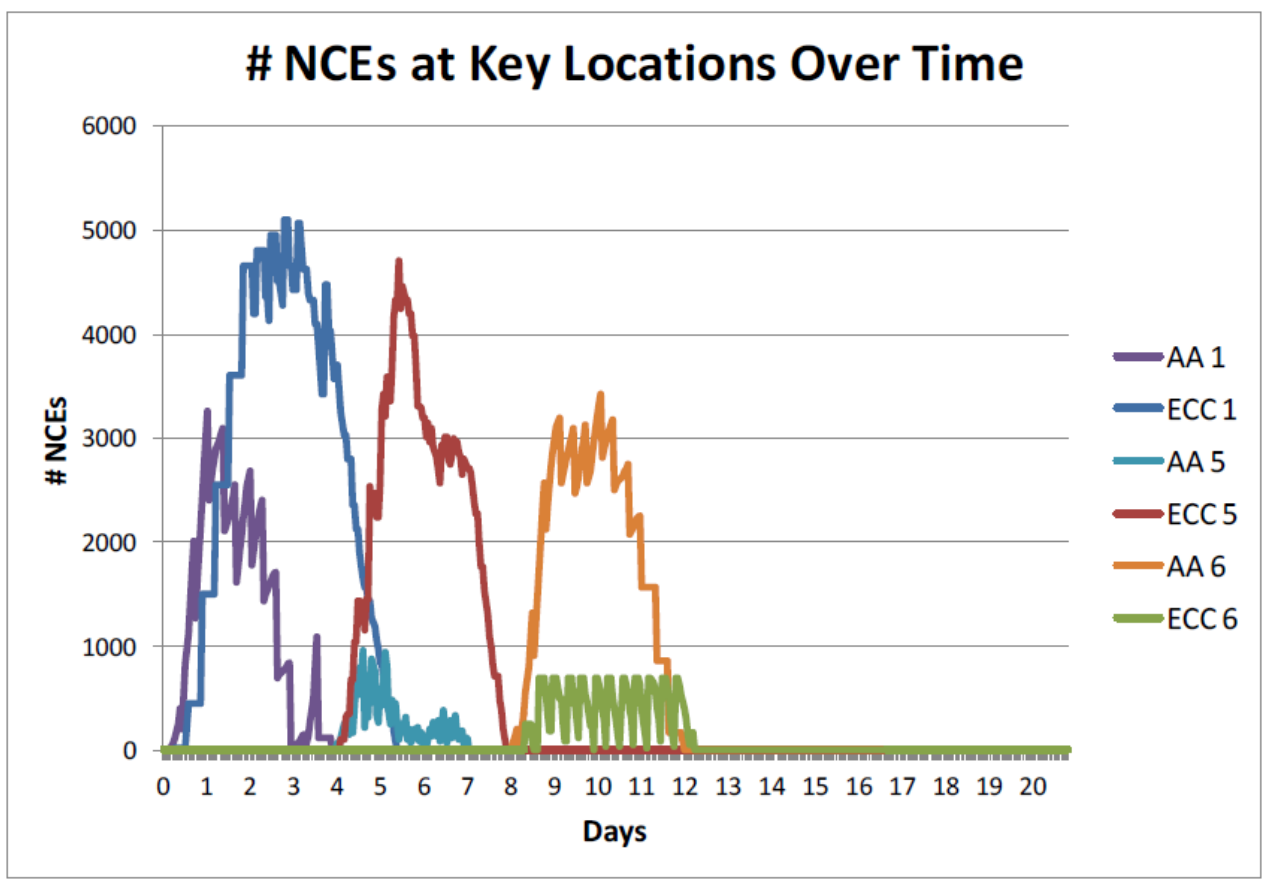

Figure 7: Key Location Contents Over Time.

\subsection{Queue Statistics}

Table 2 shows some of the statistics we use to analyze the performance of the system. First, we notice the timeframe that each location is utilized. The maximum number of NCEs at each location can be used to determine requirements for location holding capacities and highlight locations with insufficient capacity. The total number of person-days can be used to determine the amount of resources (security forces, food, water, bathrooms, tents, etc.) needed to care for NCEs at each location. Mean time in queue shows how long the average evacuee spends at each location. Long-term provisions like beds/tents may be necessary for longer durations vs. short times in queue.

By examining the mean time in queue statistic, we see that ECC1, ECC5, and AA6 have the longest queue times. These locations can be interpreted as bottlenecks in the evacuation and there is simply not enough throughput to move the NCEs fast enough to avoid significant accumulation at these locations.

We also see that AA1, ECC1, AA5, AA6, and TSH2 experience maximums of greater than 2,500 NCEs. This is a significant number of evacuees and improvements to location infrastructure, including lodging, restrooms, water, and food would need to be considered.

We also calculate the total number of person-days that each location experiences. This figure can be used to determine total resource requirements for each location over the course of the evacuation. 
Kuchel

Table 2: Summary Statistics.

\begin{tabular}{|l|r|r|r|r|r|}
\hline Location & $\begin{array}{c}\text { Mean Arrival } \\
\text { Time (hrs) }\end{array}$ & $\begin{array}{c}\text { Mean Time of } \\
\text { Last NCE Evac } \\
\text { (hrs) }\end{array}$ & $\begin{array}{c}\text { Max } \\
\text { NCEs }\end{array}$ & $\begin{array}{c}\text { Total } \\
\text { Person- } \\
\text { Days }\end{array}$ & $\begin{array}{c}\text { Mean Time in } \\
\text { Queue (hrs) }\end{array}$ \\
\hline AA1 & 0 & 86 & 3379 & 5,053 & 11.8 \\
\hline ECC1 & 6 & 130 & 2895 & 14,553 & 33.5 \\
\hline ECC2 & 0 & 79 & 308 & 801 & 17.6 \\
\hline ECC3 & 0 & 76 & 244 & 525 & 12 \\
\hline ECC4 & 0 & 73 & 280 & 618 & 13.6 \\
\hline AA5 & 96 & 169 & 1067 & 893 & 1.5 \\
\hline ECC5 & 99 & 190 & 1497 & 9,187 & 17.3 \\
\hline AA6 & 192 & 288 & 3469 & 7,608 & 22.1 \\
\hline ECC6 & 200 & 296 & 270 & 1,665 & 3.02 \\
\hline TSH1 & 9 & 303 & 2187 & 7,247 & 7.9 \\
\hline TSH2 & 113 & 203 & 2930 & 4,312 & 8.3 \\
\hline
\end{tabular}

\subsection{Excursion Analysis}

For our additional courses of action, we want to examine changing variables that will significantly improve or decrease system performance. We will consider Mean Time of Last NCE Evacuated and Max NCEs in Queue to be our primary indicators of system performance at a particular location. From the results of the base case, we find the size of the queue at AA1 and AA6 to be problematic. These locations may be in a dangerous environment and ill-suited to handle thousands of NCEs for several days. To increase the rate of evacuation, we consider a case with additional ground vehicles. We increase the number of ground vehicles at AA1 from 15 to 20 and increase the number at AA6 from 10 to 15. Table 3 shows that the maximum number of NCEs greatly decreases and the evacuation time improves at both locations.

Table 3: Increased Ground Vehicles COA.

\begin{tabular}{|l|r|r|r|r|}
\hline \multirow{2}{*}{} & \multicolumn{2}{|c|}{ Base } & \multicolumn{2}{c|}{ Increased } \\
\cline { 2 - 5 } & $\begin{array}{c}\text { Mean Time } \\
\text { Last NCE }\end{array}$ & $\begin{array}{c}\text { Max } \\
\text { NCEs }\end{array}$ & $\begin{array}{c}\text { Mean Time } \\
\text { Last NCE }\end{array}$ & $\begin{array}{c}\text { Max } \\
\text { NCEs }\end{array}$ \\
\hline AA1 & 3.92 & 3364 & 3.84 & 2619 \\
\hline AA6 & 12.1 & 3852 & 11.3 & 2387 \\
\hline
\end{tabular}

By evacuating NCEs from AA1 more rapidly, a longer queue is created at ECC1. We would like to see what changes could alleviate the increased numbers at ECC1. We will examine how various levels of C-17s will affect ECC1. The quantity of lift assets devoted to executing an operation are often a decision variable that Commander is interested in. The commander must request lift assets from TRANSCOM to execute the mission (HQDA 2005). Thorough analysis illustrating the benefits of receiving additional lift assets strengthens the Commander's request for lift.

The base case value in our prior model runs was $10 \mathrm{C}-17 \mathrm{~s}$. However, we want to consider a full range of 8-12 C-17s. C-17s are used to transport NCEs from ECCs 1, 5, and 6 and we are interested to see how various quantities of aircraft affect the evacuation of the system.

Table 4 shows that $10 \mathrm{C}-17 \mathrm{~s}$ does not offer any improvement over $8 \mathrm{C}-17 \mathrm{~s}$. The lack of improvement is likely due to MOG constraints and NTS processing delays that cause the additional lift capability to be unutilized. 
Kuchel

Table 4: C-17 Qty COA Comparison.

\begin{tabular}{|r|r|r|r|r|r|r|r|}
\hline \multicolumn{1}{|c|}{} & \multicolumn{8}{|c|}{ Mean Evacuation Day } \\
\cline { 2 - 9 } & & AA1 & ECC1 & AA5 & ECC5 & AA6 & ECC6 \\
\hline \multirow{3}{*}{ C-17s } & 8 & 3.9 & 4.9 & 7.1 & 7.9 & 10.9 & 11.2 \\
\cline { 2 - 9 } & 10 & 3.9 & 4.9 & 7.1 & 7.9 & 10.9 & 11.2 \\
\cline { 2 - 9 } & 12 & 3.9 & 4.9 & 7.1 & 7.9 & 10.9 & 11.2 \\
\hline
\end{tabular}

However, additional model runs shown in Table 5 show us that significant value can be gained by making the C-17s available on Day 1 rather than Day 2, which is the base case. Earlier availability of C$17 \mathrm{~s}$ causes the maximum number of NCEs at ECC 1 to decrease from 6,342 to 4,006. This analysis would inform the Commander to request fewer C-17s, but to request them to available on Day 1.

Table 5: C-17 Start Day COA.

\begin{tabular}{|l|r|r|r|r|r|r|}
\hline & \multicolumn{7}{|c|}{ Maximum NCEs } \\
\cline { 2 - 8 } & \multicolumn{1}{|c|}{ AA1 } & ECC1 & AA5 & ECC5 & AA6 & ECC6 \\
\hline Day 1 & 2734 & 4006 & 987 & 3564 & 1462 & 2340 \\
\hline Day 2 & 2762 & 6342 & 856 & 3984 & 1562 & 2362 \\
\hline
\end{tabular}

\subsection{Additional COA Comparison}

There are many instances of what-if analyses that are often performed on NEO scenarios. We will not evaluate alternate scenarios for the notional example, but rather discuss the types of COAs that are typically analyzed.

According to Davis (2010), many NEO planners consider a base case with 3 times the expected number of NCEs. In our experience we have found that USARAF and USPACOM were also interested in a 2.5 or 3 times population estimate. In the past, more NCEs have asked to be evacuated than was originally planned for and planners like to use this figure as an upper bound.

Airfield MOG, or maximum on the ground, is another resource that can constrain the flow of NCEs in an evacuation. MOG can be equated to the number of planes that can be on the ground at an airfield at any point in time. The US military will receive an apportionment of MOG from each airfield. However, having analysis to illustrate consequences and delays that will be incurred by having insufficient MOG can help the Commander to negotiate for sufficient airfield MOG.

NTS machines must be allocated throughout the evacuation network to process and track NCEs. Analysis can be used to inform the resource allocation decision problem and detect bottlenecks due to insufficient processing capability.

Occasionally, there are several locations that could be used as an ECC or TSH in an evacuation. The differing travel distances, MOG, and holding capacities can greatly affect evacuation times. The simulation can easily be altered to model various locations and compare results.

Many airfields in austere locations are incapable of 24 hour operations. However, the military can bring in equipment to improve the airfield to handle 24 hour operations, at least temporarily. Running certain locations for 12 vs. 24 hours easily highlights the differences and can be used to gauge the costbenefit of the investment to improve an airfield.

In the notional example described above, the evacuation took place sequentially. Area 1 was evacuated, then area 2, and finally area 3. The commander is often interested in what would happen if the evacuation timing could not be controlled and all three areas required simultaneous evacuation.

As discussed in Scheer (2011), NCE arrival patterns can vary greatly. We have been informed by DOS that most arrivals are bell shaped with a tail to the right. However, we are interested in how system performance would change if faced with a different arrival distribution. 


\section{SUMMARY}

The Center for Army Analysis has developed a methodology for quickly modeling Noncombatant Evacuation Operations and analyzing courses of action to inform Army decision makers. We are able to model individual NCEs, lift assets, and resources throughout the operation and provide insights into evacuation times and constraining factors. The model can help determine necessary resource levels given a targeted evacuation time or can determine an evacuation time given fixed resource levels. Decision makers are able to use this information to determine requirements for security forces, food, water, and shelter and better prepare and provide for American citizens in the event of an evacuation.

\section{FUTURE WORK}

Going forward, we would like to incorporate simulation optimization techniques to help inform decisions like allocating NTS machines. Arena has a built in function that will conduct many simulation runs, changing specified variables each time, attempting to minimize evacuation time. NTS allocation becomes more complex when the evacuation is tiered and the machines can be moved to new locations. However, an optimization will be an improvement over manual calculation and excursion simulation trials.

We will also continue to improve the modeling methodology to improve modeling time and produce faster results for the next scenario of interest. The NEO process demands quick turn analysis and Commanders are more interested in faster results than more detailed statistical analysis.

\section{REFERENCES}

Davis, A. M. 2007. "Joint Consideration for Planning and Conducting NEOs". Joint Military Operations Department, Naval War College.

Ford, T. J. 2007. "State Department: The July 2006 Evacuation of American Citizens from Lebanon". United States Government Accounting Office.

Gregg, N. A. 2010. "Optimizing Crisis Action Planning In The Noncombatant Evacuation Operation Setting”. Air Force Institute of Technology.

Headquarters, Department of the Army. 200. "Joint Plan for DoD Noncombatant Evacuation and Repatriation". Headquarters, Department of the Army.

Joint Chiefs of Staff. 2007. "Noncombatant Evacuation Operations". Joint Publication 3-68.

Junkins, L. J. 2012. "Noncombatant Evacuation Operations (NEO) Decision-Making Process Effects on Efficiency". Air War College, Air University.

Kelton, W.D., R.P. Sadowski, and D.T. Sturrock. 2007. Simulation with ARENA, Fourth Edition. New York, NY: The McGraw-Hill Companies, Inc.

Scheer, A. M. 2011. "Noncombatant Evacuation Operations in EUCOM". Air Force Institute of Technology.

State Department. 2013. "Drawdown / Evacuation - U.S. Diplomatic Posts Under Authorized and Ordered Departure Authority". Leadership and Management School Foreign Service Institute.

\section{AUTHOR BIOGRAPHY}

DALLAS KUCHEL has been an Operations Research Analyst at the Center for Army Analysis since 2010. He graduated with distinction from the University of Virginia with degrees in Systems Engineering and Economics in 2010. He is currently pursuing a Masters degree in Operations Research from George Mason University. His interests include applying analytical techniques such as simulation, optimization, and decision analysis to solve real world problems. He is an avid sports fan and is known to apply analytical techniques to dominate his fantasy football and baseball leagues. His email address is dallas.kuchel.civ@mail.mil. 